\title{
Construction of Solutions with Exactly k Blow-up Points for the Schrödinger Equation with Critical Nonlinearity
}

\section{Frank Merle}

Centre de Mathématiques Appliquées, Ecole Normale Supérieure, 45, rue d'Ulm, F-75230 Paris, Cedex 05, France

Abstract. We consider the nonlinear Schrödinger equation:

$$
i \partial u / \partial t=-\Delta u-|u|^{4 / N} u \quad \text { and } \quad u(0, .)=\varphi(.)
$$

where $u:[0, T) \times \mathbb{R}^{N} \rightarrow \mathbb{C}$. For any given points $x_{1}, x_{2}, \ldots, x_{k}$ in $\mathbb{R}^{N}$, we construct a solution of Eq. (1), $u(t)$, which blows up in a finite time $T$ at exactly $x_{1}, x_{2}, \ldots, x_{k}$. In addition, we describe the precise behavior of the solution $u(t)$ when $t \rightarrow T$, at the blow-up points $\left\{x_{1}, x_{2}, \ldots, x_{k}\right\}$ and in $\mathbb{R}^{N}-\left\{x_{1}, x_{2}, \ldots, x_{k}\right\}$.

\section{Introduction and Main Results}

In the present paper, we consider the Schrödinger equation:

$$
i \partial u / \partial t=-\Delta u-|u|^{p-1} u \quad \text { and } \quad u(0, .)=\varphi(.)
$$

where $\Delta$ is the Laplace operator on $\mathbb{R}^{N}, u:[0, T) \times \mathbb{R}^{N} \rightarrow \mathbb{C}, p=1+4 / N$, and $\varphi \in H^{1}\left(\mathbb{R}^{N}\right)$. More precisely, we say that $u($.$) is a solution of Eq. (1) on [0, T)$ if $\forall t \in[0, T)$,

$$
u(t)=S(t) \varphi+i \int_{0}^{t} S(t-s)\left\{|u(s)|^{4 / N} u(s)\right\} d s
$$

where $S($.$) is the group with infinitesimal generator i \Delta$ (the Schrödinger group) and for each $t, u(t)$ denotes the function $x \rightarrow u(t, x)$.

For $p \in\left(1,2^{*}-1\right)$ (where $2^{*}=2 N /(N-2)$ if $N>2$, otherwise $\left.2^{*}=+\propto\right)$, it is well known that Eq. (1) has a unique solution $u(t)$ in $H^{1}$ and there exists $T>0$ such that $\forall t \in[0, T), u(t) \in H^{1}$ and either $T=+\propto$ or $\lim _{t \rightarrow T}\|u(t)\|_{H^{1}}=+\propto$ (see Ginibre and Velo [4,5], Kato [7]). Furthermore, we have $\forall t \in[0, T)$,

$$
\begin{gathered}
\|u(t)\|_{L^{2}}=\|\varphi\|_{L^{2}}, \\
E(u(t))=(1 / 2)\|\nabla u(t)\|_{L^{2}}^{2}-(1 /(p+1)) \int|u(t, x)|^{p+1} d x=E(\varphi) .
\end{gathered}
$$


When $p=1+4 / N$, we have

$$
\begin{aligned}
(d / d t) \int|x|^{2}|u(t)|^{2} & =4 \operatorname{Im} \int r u(t, x) \bar{u}_{r}(t, x) d x, \\
\left(d^{2} / d t^{2}\right) \int|x|^{2}|u(t)|^{2} & =8 E(\varphi), \quad \text { where } r=|x| \text { and } u_{r}=\partial u / \partial r
\end{aligned}
$$

For $p<1+4 / N$, the conservation of the energy (3) implies that blowing-up in finite time never occurs (Ginibre and Velo [4]). On the other hand, it is well known that for $p \geqq 1+4 / N$, there are singular solutions of Eq. (1) for suitable initial data (see Zakharov, Sobolev and Synach [17], Glassey [6]). That is, there exist solutions $u(t)$ of Eq. (1) such that $u(.) \in C\left([0, T), H^{1}\right)$ and $\lim _{t \rightarrow T}\|u(t)\|_{H^{1}}=\left|\lim _{t \rightarrow T}\right|\|u(t)\|_{L^{2}+4 / N}=$ $+\propto$. Our aim is to have a better understanding of the blow-up solutions' behavior and in particular of the local behavior of these solutions, in the critical power case $p=1+4 / N$. For $N=2$, this model has a physical interest: it can be considered as a first approximation to a model of a planar laser beam which is propagating along a single direction $t$ in $\mathbb{R}^{3}$.

The phenomena which occur in the case where $p=1+4 / N$ seem to be quite different from those where $p>1+4 / N$.

Indeed, in the supercritical case $1+4 / N<p<(N+2) /(N-2)$, numerical computations (Lemesurier, Papanicolaou, C. and P. L. Sulem [9]) and some mathematical analysis (Merle [11]) suggest that every blow-up solution has a strong limit in $L^{2}$ at the blow-up time.

In contrast, for $p=1+4 / N$ Merle and Tsutsumi ([13]) show that a blow-up solution never has a strong limit in $L^{2}$ at the blow-up time. In addition, if the initial data $\varphi$ has a spherical symmetry, then an $L^{2}$-concentration phenomenon occurs at the origin at the blow-up time (see also [16,8]). More precisely, for all $R>0$, we have $\liminf _{t \rightarrow T}\|u(t)\|_{L^{2}(B(0, R))} \geqq\|Q\|_{L^{2}}$, where $T$ is the blow-up time and $Q$ is the ground state solution of the equation

$$
-\Delta u+u-|u|^{4 / N} u=0
$$

(for the existence of the function $Q$, see for example Weinstein [15]).

In this paper, we are interested in the local behavior of the solution of Eq. (1) at the blow-up time and in particular we are interested in what the blow-up sets $B_{H^{1}}$ and $B_{L^{2+4 / N}}$ look like.

Let us first define the blow-up set $B_{F}$ for a functional space $F$.

Definition. Assume that there exists $T>0$ such that $\forall t \in[0, T), u(t) \in H^{1}$ and $\lim \|u(t)\|_{H^{1}}=+\propto$. We denote by $B_{F}$ the set of points $x_{0}$ such that for all $R>0$, $t \rightarrow T$

there is a sequence $t_{n} \rightarrow T$ such that $\left\|u\left(t_{n}\right)\right\|_{F\left(B\left(x_{0}, R\right)\right)} \rightarrow+\propto$, where $\|v\|_{F\left(B\left(x_{0}, R\right)\right)}$ is the norm in $F$ of the restriction of $v$ at the ball of center $x_{0}$ and radius $R$.

Nothing is really known about these sets. For example, we do not know if $B_{H^{1}}=B_{L^{2+4 / N}}$. In the particular case where $p=1+4 / N$, we have more information on Eq. (1). Equation (1) has a pseudoconformal invariance law; if $u(t, x)$ is a solution of Eq. (1), then $|t|^{-N / 2} e^{\left(i|x|^{2}\right) / 4 t} \bar{u}(1 / t, x / t)$ is again a solution of (1) (see [14]). This invariance law yields explicit blow-up solutions:

$$
u(t, x)=|T-t|^{-N / 2} e^{\left[(-i /(T-t))+\left(i|x|^{2}\right) / 4(T-t)\right]} R(x /(T-\mathrm{t})),
$$


where $R$ is a stationary solution of Eq. (1) in the sense where $R$ satisfies the following Eq. (5) (see [2]). These are the only explicitly known blow-up solutions. In this case, we check that

- the origin 0 is the only blow-up point,

-for all $R>0, \lim _{t \rightarrow T}\|u(t)\|_{L^{2}(B(0, R))}=\|\varphi\|_{L^{2}}$ $-\int|x|^{2}|u(t, x)|^{2} \rightarrow 0$ as $t \rightarrow T$.

Therefore it is natural to ask if there exist blow-up solutions of Eq. (1) such that the set of blow-up points is different from a single point and what behavior we can expect at such points.

Our result is the following: for any given points $x_{1}, x_{2}, \ldots, x_{k}$ in $\mathbb{R}^{N}$, we construct a solution $u(t)$ of Eq. (1) which blows-up in finite time $T$ at exactly $x_{1}, x_{2}, \ldots, x_{k}$. In addition, we have the precise behavior of $u(t)$ at the points $x_{i}$, for $i=1, \ldots, k$. We prove for these particular solutions the existence of an $L^{2}$-concentration phenomenon at the points $x_{i}$ : for all $R>0$, and for $i=1, \ldots, k$, we have $\lim \|u(t)\|_{L^{2}\left(B\left(x_{i}, R\right)\right)}=\left\|Q_{i}\right\|_{L^{2}}$, where for $i=1, \ldots, k, Q_{i}$ is a solution different from $\rightarrow \rightarrow T$ zero of Eq. (5). More precisely, we have the following result:

Theorem. Let $x_{1}, x_{2}, \ldots, x_{k}$ in $\mathbb{R}^{N}$ and $Q_{1}, \ldots, Q_{k}$ be radially symmetric solutions of Eq. (5).

Then there is a constant $\omega_{0}$ such that for $\omega_{1}>\omega_{0}, \ldots, \omega_{k}>\omega_{0}$, there exists a solution $u(t)$ of Eq. (1) which blows-up in finite time T and such that

- the set of blow-up points in $L^{2+4 / N}$ and $H^{1}$ is $\left\{x_{1}, x_{2}, \ldots, x_{k}\right\}$,

- for $i=1, \ldots, k$, and all $R>0$ such that the balls $B\left(x_{i}, R\right)$ are disjoint,

$$
\lim _{t \rightarrow T}\|u(t)\|_{L^{2}\left(B\left(x_{i}, R\right)\right)}=\left\|Q_{i}\right\|_{L^{2}}
$$

- for all $R>0, \lim _{t \rightarrow T}\|u(t)\|_{L^{2}}\left(\mathbb{R}^{N} \backslash \bigcup_{i=1, \ldots, k} B\left(x_{i}, R\right)\right)=0$.

In addition, there is a constant $\gamma>0$ such that

$$
\begin{aligned}
& \left\|u(t)-\left\{\sum_{i=1}^{i=k}\left|(T-t) \omega_{i}\right|^{-N / 2} e^{\left(-t /(T-t) \omega_{t}^{2}\right)+\left(|||x|^{2}\right) / /(T-t)} Q_{i}\left(\left(x-x_{i}\right) /(T-t) \omega_{i}\right)\right\}\right\|_{L^{2+4 / N}} \\
& \leqq e^{-\gamma /|T-t|}
\end{aligned}
$$

on $[0, T)$.

Remark 0 . Schoen has obtained similar results for elliptic equations with critical power in $\left[13^{\prime}\right]$.

Remark 1. The main point of the theorem is fact that the result is true for any given points $x_{1}, x_{2}, \ldots, x_{k}$ in $\mathbb{R}^{N}$.

Remark 2. We only need that the functions $Q_{i}$ are solutions of Eq. (5) and decrease exponentially at infinity. 
Remark 3. We remark that the behavior of the solution $u(t)$ obtained in the theorem at the blow-up time is the same (except for the number of blow-up points) as the behavior of the explicit blow-up solutions (see above).

There is a concentration of mass (in the norm $L^{2}$ ) at the blow-up points $x_{1}, x_{2}, \ldots, x_{k}$. Since for all solutions of Eq. (5), $Q^{\prime}$ different from zero, we have $\left\|Q^{\prime}\right\|_{L^{2}} \geqq\|Q\|_{L^{2}}$ (by definition of the ground state); for all $R>0$ and $i=1, \ldots, k$, we have $\liminf _{t \rightarrow T}\|u(t)\|_{L^{2}\left(B\left(x_{i}, R\right)\right)} \geqq\|Q\|_{L^{2}}$. This result is connected to the one obtained by Merle and Tsutsumi in [13]: if $\varphi$ has a spherical symmetry, for all $R>0$, we have $\liminf \|u(t)\|_{L^{2}(B(0, R))} \geqq\|Q\|_{L^{2}}$ (where $T$ is the blow-up time).

On the other hand, at the blow-up time all the mass concentrate around the blow-up points. That is for all $R>0, \lim _{t \rightarrow T}\|u(t)\|_{L^{2}}\left(\mathbb{R}^{N} \backslash \bigcup_{i=1, \ldots, k} B\left(x_{i}, R\right)\right)=0$.

Remark 4. For the heat equation in dimension one on $[0,1]$,

$$
\partial u / \partial t=\Delta u+|u|^{p-1} u \text { on }[0,1] \text { and } u(0)=u(1)=0,
$$

Chen and Matano ([10]) show that when the blowing-up in finite time occurs, there is only a finite number of blow-up points. Open problems are first to show the existence of a solution which blows up at exactly $k$ points, and whether or not we can choose these $k$ blow-up points arbitrarily.

An important open problem is to find for any blow up solution what the blow-up set looks like. We can expect that the blow-up set is a finite union of manifolds. In the special case of an initial data $\varphi$ with spherical symmetry, consider the solution of the Schrödinger equation, $u(t)$ and assume that $u(t)$ blows up in finite time. Can we show that the origin is the only blow-up point in $H^{1}$.

The theorem has the following corollary.

Corollary 1. It is false in general that at the blow-up time, we have

$$
\lim _{t \rightarrow T}\left\{\inf _{y \in \mathbb{R}^{N}} \int|x-y|^{2}|u(t, x)|^{2} d x\right\}=0 .
$$

On the other hand, the theorem has a "dual" version. Indeed, we now remark that using the conformal invariance law of Eq. (1) on the solution obtained in the theorem yields the corollary

Corollary 2. Let $Q_{1}, \ldots, Q_{k}$ be radially symmetric solutions of Eq. (5). Then for $\omega>0$, there is an initial data $\varphi$ such that the solution of Eq. (1) $u(t)$ is globally defined. In addition, there exist $y_{1}(),. \ldots, y_{k}(.) \in C\left(\mathbb{R}, \mathbb{R}^{N}\right), \omega_{1}(),. \ldots, \omega_{k}(.) \in C\left(\mathbb{R}^{N+1}, \mathbb{R}\right)$ such that

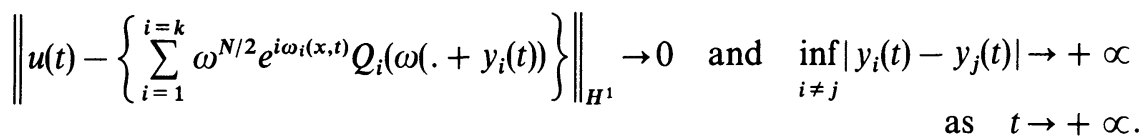

Remark 5. As before, we only need the exponential decay of functions $Q_{i}$ at infinity.

Remark 6. Therefore we obtain a solution which behaves like a sum of periodic functions travelling with different constant speed. Of course using rescaling 
arguments on the functions $Q_{i}$, we can obtain a function $u(t)$ which behaves like a sum of periodic functions with different periods. Similar results can be also proved with some type of $k$-coupled nonlinear Schrödinger equations.

Moreover there is an application to the Cauchy problem of Eq. (1). Indeed, we have

Corollary 3. Let $x_{1}, x_{2}, \ldots, x_{k}$ in $\mathbb{R}^{N}$ and $Q_{1}, \ldots, Q_{k}$ be radially symmetric solutions of Eq. (5). Then there is a solution $u(t)$ of Eq. (1) such that $u(t)$ is solution of (1) on $(0, T)$ for $T>0$, and

$$
|u(t)|^{2} \rightarrow \sum_{i=1}^{i=k}\left|Q_{i}\right|_{L^{2}}^{2} \delta_{x=x_{i}} \text {, when } t \rightarrow 0 .
$$

In fact, there is a $k$ parameters' family of solutions.

The interest of this paper is twofold.

i) We construct explicit examples of blow-up solutions with a blow-up set different from a point.

ii) We introduce a new method to construct solution of Eq. (1). We proceed as follows. We remark that the function

$$
Q_{T}(t)=\sum_{i=1}^{i=k}|T-t|^{-N / 2} e^{-i /(T-t)+\left(i|x|^{2}\right) / 4(T-t)} Q_{i}\left(\left(.-x_{i}\right) /(T-t)\right)
$$

is almost a solution of Eq. (1), since it is a sum of functions which are "decoupled," at time $T$. Therefore with techniques of a priori estimates on exact solution of Eq. (1) with one approximate solutions of Eq. (1) and compactness arguments, we construct a solution which behaves like $Q_{T}(t)$ at time $T$.

The paper is organized as follows:

In Sect. 2, we give a precise outline of the method.

In Sect. 3, we establish crucial a priori estimates on a class of solutions of Eq. (1).

Section 4 is devoted to the proof of compactness results.

In Sect. 5, we finally prove our main result.

We conclude this section by giving several notations. We abbreviate $L^{q}\left(\mathbb{R}^{N}\right)$ and $\|\cdot\|_{L^{q\left(\mathbb{R}^{N}\right)}}$ by $L^{q}$ and $\|\cdot\|_{L^{q}}$. We put $\sigma=2(N+2) / N=(1+4 / N)+1$. For $s>1$, we define $\bar{s}$ such that $1 / s+1 / \bar{s}=1$.

\section{Outline of the Proof the Theorem}

Let $x_{1}, x_{2}, \ldots, x_{k}$ be in $\mathbb{R}^{N}$, and $Q_{1}, \ldots, Q_{k}$ be radially symmetric solutions of Eq. (5). For convenience, using the autonomous character of Eq. (1), we have to find an initial data $\varphi, \omega>0$ and an $a>0$ such that the solution $u(t)$ of the following equation:

$$
u(-a)=\varphi \quad \text { and } \quad i \partial u / \partial t=-\Delta u-|u|^{4 / N} u,
$$

blows-up at time 0 such that

-the sets of blow-up points in $H^{1}$ and $L^{2+4 / N}$ is $\left\{x_{1}, x_{2}, \ldots, x_{k}\right\}$,

-for $i=1, \ldots, k$, and small $R>0, \lim _{t \rightarrow T}\|u(t)\|_{L^{2}\left(B\left(x_{i}, R\right)\right)}=\left\|Q_{i}\right\|_{L^{2}}$, 
-for all $R>0, \lim _{t \rightarrow T}\|u(t)\|_{L^{2}\left(\mathbb{R}^{N} \backslash \underset{i=1, \ldots, k}{\bigcup} B\left(x_{i}, R\right)\right)}=0$, $-\left\|u(t)-\left\{\sum_{i=1}^{i-k}|(T-t) \omega|^{-N / 2} e^{\left(-i /(T-t) \omega^{2}\right)+\left(i|x|^{2}\right) / 4(T-t)} Q_{i}\left(\left(x-x_{i}\right) /((T-t) \omega)\right)\right\}\right\|_{L^{2+4 / N}} \leqq$
$e^{-\gamma /|T-t|}$ on $[0, T)$.

The proof in the general case will be the same.

Let us consider the function $Q_{T}(t)=\sum_{i=1}^{i=k}|t|^{-N / 2} e^{-i / t+i|x|^{2} / 4 t} Q_{i}\left(\left(.-x_{i}\right) / t\right)$, for $t<0$ $(\omega=1)$. We first remark that $Q_{T}(t)$ has the behavior we want at the blow-up time. Unfortunately, it is not an exact solution of the Eq. (6), but a computation yields that it is "almost" a solution of Eq. (6) for $t$ near zero. In fact, there is a competition between two phenomena. Indeed, for a fixed $i$, the function $|t|^{-N / 2} e^{i / t+i|x|^{2} / 2 t} Q_{i}\left(\left(-x_{i}\right) / t\right)$ is concentrated at the point $x_{i}$ as $t \rightarrow 0$ and is decoupled with the others. On the other hand for $t \rightarrow 0$, the function $|t|^{-N / 2} e^{i / t+i|x|^{2} / 2 t} Q_{i}\left(\left(.-x_{i}\right) / t\right)$ goes out of space $H^{1}$, where we solve the Cauchy problem of the Schrödinger equation. Our aim is to find a solution $u(t)$ of Eq. (6) such that $\forall t \in[-a, 0)$, we have $\left\|u(\bar{t})-Q_{T}(t)\right\|_{L^{\sigma}} \leqq e^{-\gamma /|t|}$ (where $\gamma$ is a constant which does not depend on $t$ ). We then verify that a function $u(t)$ which satisfies such a property has the behavior we want at time zero. The fact that the solutions $Q_{i}$ and the blow-up phenomena are strongly unstable ([3]) imply that it is difficult to have the same local blow-up time at points $x_{1}, \ldots, x_{k}$. To illustrate this fact, for example, we do not obtain a solution with the behavior we want by taking for $\varepsilon>0$ and small, the solution of the equation

$$
u(-\varepsilon)=Q_{T}(-\varepsilon) \quad \text { and } \quad i \partial u / \partial t=-\Delta u-|u|^{4 / N} u .
$$

Rather, we use two steps to find such a solution $u(t)$. We consider $u_{\varepsilon}($.$) , the$ solution of the following problem:

$$
u(-\varepsilon)=Q_{T}(-\varepsilon) \quad \text { and } \quad i \partial u / \partial t=-\Delta u-|u|^{4 / N} u .
$$

In a first step, for a fixed number $a>0$, we seek estimates on $u_{\varepsilon}(t)$ for $t \in[-a,-\varepsilon)$ uniformly on $\varepsilon$. For this purpose, we work in the space $L^{\sigma}$ which is a natural space in which to solve the Cauchy problem of Eq. (1). We obtain these estimates using a fixed point formulation in $L^{\sigma}$ of equation $(\varepsilon)$ and techniques of a priori estimates.

In the second step, using the conservation law (2)-(4), we obtain compactness results on the set $\left\{u_{\varepsilon}(-a)\right\}$. Lastly, we verify that the solution $u(t)$ of Eq. (1) obtained with an initial data $\varphi$ such that there is a sequence $\varepsilon_{n} \rightarrow 0$ such that $u_{\varepsilon_{n}}(-a) \rightarrow \varphi$ when $n \rightarrow+\propto$, has the behavior we want at blow-up time.

\section{A Priori Estimates}

In this section, for a number $a>0$, we estimate $u_{\varepsilon}(t)$ on $[-a,-\varepsilon]$ when $\varepsilon \rightarrow 0$ and more precisely the quantity $u_{\varepsilon}(t)-Q_{T}(t)=u_{\varepsilon}(t)-\left\{\sum_{i=1}^{i=k}|t|^{-N / 2} e^{-i / t+\left(i|x|^{2}\right) / 4 t} Q_{i}\left(\left(.-x_{i}\right) / t\right)\right\}$, when inf $\left|x_{i}-x_{j}\right|$ is large. This assumption is technical and has no consequences $i \neq j$

on the conclusions of the theorem. Our main tools are, on one hand, the fixed 
point formulation on $L^{\sigma}$ of equation $(\varepsilon)$ and the fact that $Q_{T}(t)$ is "almost" a solution of Eq. (1) for $t$ near the "blow-up time."

We use a priori estimates techniques. We remark $([14])$ that there is a constant $\alpha>0$ such that $\forall t \in[-1,0),\left\|Q_{T}(t)\right\|_{L^{\sigma}} \leqq \alpha /|t|^{2 / \sigma}$. Therefore, we want to prove that for a fixed $\varepsilon$,

if for $s \in[t,-\varepsilon),\left\|u_{\varepsilon}(s)\right\|_{L^{\sigma}} \leqq(\alpha+1) /|s|^{2 / \sigma}$, we have

$\left\|u_{\varepsilon}(t)-Q_{T}(t)\right\|_{L^{\sigma}} \leqq e^{-\gamma /|t|}$ (where $\gamma$ is a constant which does not depend on $\varepsilon$ ).

Proposition 1. Let $Q_{1}, \ldots, Q_{k}$ be radial symmetric solutions of Eq. (5) with a finite number of zeros (in spherical coordinates).

i) There exists a constant $\beta_{Q_{i}, k}>0$ such that for $\inf _{i \neq j}\left|x_{i}-x_{j}\right| \geqq \beta_{Q_{i}, k}$, there is an $\varepsilon_{0}$ such that for all $-\varepsilon_{0} \leqq t \leqq-\varepsilon$,

$\forall s \in(t,-\varepsilon),\left\|u_{\varepsilon}(s)\right\|_{L^{\sigma}} \leqq(\alpha+1) /|s|^{2 / \sigma}$ implies that $\left\|u_{\varepsilon}(t)-Q_{T}(t)\right\|_{L^{\sigma}} \leqq e^{-\gamma /|t|}$ (where $\gamma$ is a nonnegative constant which does not depend on $\varepsilon)$.

ii) Assume that $\inf \left|x_{i}-x_{j}\right| \geqq \beta_{Q_{i}, k}$. There exist $a>0, K>0, \gamma>0$ such that for $\varepsilon \in\left(0, \varepsilon_{0}\right]$ we have $\left\|u_{\varepsilon}(-a)\right\|_{L^{\sigma}} \leqq K$, and for $t \in[-a,-\varepsilon],\left\|u_{\varepsilon}(t)-Q_{T}(t)\right\|_{L^{\sigma}} \leqq e^{-\gamma /|t|}$.

Let us first establish some lemmas and preliminary results.

Lemma 1. i) Assume that $u \in L^{\bar{\sigma}}$, then $\forall t \neq 0, S(t) u \in L^{\sigma}$ and $\|S(t) u\|_{L^{\sigma}} \leqq c|t|^{-1 /(1+2 / N)}\|u\|_{L^{\tilde{j}}}$ (where $c$ does not depend on $t$ and $u$ ).

ii) Assume that $u \in L^{2}$, then $\forall t S(t) u \in L^{2}$ and $\|S(t) u\|_{L^{2}}=\|u\|_{L^{2}}$.

Proof. See Ginibre and Velo [4].

Lemma 2. Assume that $u$ and $v$ belong to $L^{\sigma}$. Then we have for $t \neq 0$,

$$
\left\|S(t)\left\{|u|^{4 / N} u-|v|^{4 / N} v\right\}\right\|_{L^{\sigma}} \leqq c|t|^{-1 /(1+2 / N)}\left\{\|u\|_{L^{\sigma}}^{4 / N}+\|v\|_{L^{\sigma}}^{4 / N}\right\}\|u-v\|_{L^{\sigma}} .
$$

Proof. See Ginibre and Velo [4].

We state a simple result on solutions of Eq. (5) which is fundamental in the proof of the theorem.

Lemma 3. Let be $Q$ a radial solution of Eq. (5) with a finite number of zeros (in spherical coordinates). Then there are constants $c>0$ and $\theta>0$ such that

$$
\forall r>0, \quad|Q(r)|+\left|Q^{\prime}(\dot{r})\right| \leqq c e^{-\theta r} .
$$

Proof. This is a classical result. In the case where $Q$ has no zero, we refer to [1] (Berestycki, Lions and Peletier). The proof in the general case is exactly the same.

As a corollary, we have the following lemma:

Lemma 4. Let be $R>0$ and $Q_{1}$ and $Q_{2}$ radial solutions of $E q$. (5) with a finite number of zero (in spherical coordinates).

i) Then $\left\|e^{-i /|t|+i|x| / 4|t|} Q(x /|t|)\right\|_{L^{\sigma}(|x| \geqq R)} \rightarrow 0$ and $\left\|e^{i /|t|+i|x|^{2} /|t|} Q(x /|t|)\right\|_{H^{1}(|x| \geqq R)} \rightarrow 0$ as $t \rightarrow 0$.

ii) Let $s \geqq 1$. There exists a constant $c>0$ such that for $t$ near zero

$$
\begin{gathered}
\left\|Q_{1}\left(\left(.-x_{1}\right) / t\right) Q_{2}\left(\left(.-x_{2}\right) / t\right)\right\|_{L^{s}}<c e^{-c\left|x_{1}-x_{2}\right| /|t|}, \\
\left\|Q_{1}^{4 / N}\left(\left(.-x_{1}\right) / t\right) Q_{2}\left(\left(.-x_{2}\right) / t\right)\right\|_{L^{s}}<c e^{-c / x_{1}-x_{2}|/| t \mid}
\end{gathered}
$$


and

$$
\left\|Q_{1}\left(\left(.-x_{1}\right) / t\right) Q_{2}^{4 / N}\left(\left(.-x_{2}\right) / t\right)\right\|_{L^{s}}<c e^{-c\left|x_{1}-x_{2}\right| /|t|} .
$$

Proof. From Lemma 3, some calculations yields

$$
\left\|e^{-\left.i|| t|+i| x\right|^{2} / 4|t|} Q(x /|t|)\right\|_{L^{\sigma}(|x| \geqq R)} \leqq e^{-R \theta /|t|} /|t|^{N} \text { and part i) follows. }
$$

Let $s \geqq 1$ and $t>0$. We have now to estimate the quantity $\| Q_{1}\left(\left(.-x_{1}\right) / t\right)$. $Q_{2}((.-x) / t) \|_{L^{s}}$. Lemma 3 implies the existence of constants $c>0$ and $\theta>0$ such that $\forall r>0,\left|Q_{1}(r)\right| \leqq c e^{-\theta r}$ and $\left|Q_{2}(r)\right| \leqq c e^{-\theta r}$. Therefore we have also $\forall r>0$, $\left|Q_{1}(r)\right|^{s} \leqq c e^{-\theta r}$ and $\left|Q_{2}(r)\right|^{s} \leqq c e^{-\theta r}$.

Hence we have

$$
\begin{aligned}
\left\|Q_{1}\left(\left(.-x_{1}\right) / t\right) Q_{2}\left(\left(.-x_{2}\right) / t\right)\right\|_{L^{s}}^{s} & \leqq \int\left|Q_{1}\left(\left(x-x_{1}\right) / t\right)\right|^{s}\left|Q_{2}\left(\left(x-x_{2}\right) / t\right)\right|^{s} d x \\
& \leqq c \int \exp \left\{-\theta\left\{\left|x-x_{1}\right|+\left|x-x_{2}\right|\right\} / t\right\} d x .
\end{aligned}
$$

On ther other hand, we have the following inequality $\left|x-x_{1}\right|+\left|x-x_{2}\right| \geqq$ $(1 / 2)\left\{\left|x-\left(x_{1}+x_{2}\right) / 2\right|+\left|x_{1}-x_{2}\right|\right\}$. Thus some computation yields

$$
\begin{aligned}
\left\|Q_{1}\left(\left(.-x_{1}\right) / t\right) Q_{2}\left(\left(.-x_{2}\right) / t\right)\right\|_{L^{s}}^{s} \leqq & c \exp \left\{(-\theta / t)\left|x_{1}-x_{2}\right|\right\} \\
& \cdot \int \exp \left\{(-\theta / t)\left|x-1 / 2\left(x_{1}+x_{2}\right)\right|\right\} d x \\
\leqq & \exp \left\{(-\theta / t)\left|x_{1}-x_{2}\right|\right\} \int \exp \{(-\theta / t)|x|\} d x \\
\leqq & c|t|^{N} \exp \left\{(-\theta / t)\left|x_{1}-x_{2}\right|\right\} .
\end{aligned}
$$

With a similar proof, we can obtain the two last estimates and Lemma 4 is proved.

Lemma 3 yields exponential estimates for $t$ near zero on the function

$$
\left|Q_{T}(t, x)\right|^{4 / N} Q_{T}(t, x)-\left\{\left.\sum_{i=1}^{i=k}|t|^{-N / 2} e^{-i / t+\left(i|x|^{2}\right) / 4 t}|| t\right|^{-N / 2} Q_{i}\left(\left.\left(.-x_{i} / t\right)\right|^{4 / N} Q_{i}\left(\left(.-x_{i}\right) / t\right)\right\}\right.
$$

which allows us to find a good estimates on $u_{\varepsilon}(t)$ with the fixed point formulation of Eq. $(\varepsilon)$. More precisely, we have

Lemma 5. Let us assume that the assumptions of the theorem are satisfied.

i) There exist $c>0$ and $\gamma>0$ such that we have for $t \in[-1,0)$,

$$
\begin{aligned}
& \left\|\left|Q_{T}(t)\right|^{4 / N} Q_{T}(t)-\left\{\left.\left.\sum_{i=1}^{i=k}|t|^{-N / 2} e^{(-i / t)+\left(i|x|^{2}\right) / 4 t}|| t\right|^{-N / 2} Q_{i}\left(\left(.-x_{i}\right) / t\right)\right|^{4 / N} Q_{i}\left(\left(.-x_{i}\right) / t\right)\right\}\right\|_{L^{\bar{\sigma}}} \\
& \quad \leqq c e^{-\gamma / t \mid} .
\end{aligned}
$$

ii) Then there exist $c$ and $\gamma$ such that we have for $t \in[-1,0)$,

$$
\begin{aligned}
& \left|\left\|\sum_{i=1}^{i=k}|t|^{-N / 2} e^{(-i / t)+\left(i|x|^{2}\right) / 4 t} Q_{i}\left(\left(.-x_{i}\right) / t\right)\right\|_{H^{1}}-\sum_{i=1}^{i=k}\left\||t|^{-N / 2} e^{(-i / t)+\left(i|x|^{2}\right) / 4 t} Q_{i}\left(\left(.-x_{i}\right) / t\right)\right\|_{H^{1}}\right| \\
& \quad \leqq c e^{-\gamma /|t|} .
\end{aligned}
$$

iii) In addition, assume that $k$ and the function $Q_{i}$ are fixed. Then $\gamma$ goes to infinity when inf $\left|x_{i}-x_{j}\right|$ goes to infinity. 
Proof. The proof is based on the exponential decay of the functions $Q_{i}$ at infinity and on the concentration phenomenon at the point $x_{i}$ of the function $Q_{i}\left(\left(.-x_{i}\right) / t\right)$ when $t$ goes to zero. This proof is somewhat technical. For simplicity, we consider the case of $k=2$, the proof in the general case will be the same.

Let us consider the quantity

$$
\begin{aligned}
A= & \left.\left.\left\{\left.|| t\right|^{-N / 2}\left(Q_{1}\left(\left(.-x_{1}\right) / t\right)+Q_{2}\left(.-x_{2}\right) / t\right)\right)\right|^{4 / N}\left(|t|^{-N / 2}\left\{Q_{1}\left(\left(.-x_{1}\right) / t\right)+Q_{2}\left(.-x_{2}\right) / t\right)\right\}\right\} \\
& -\left\{\left.\left.|t|^{-N / 2}|| t\right|^{-N / 2} Q_{1}\left(\left(.-x_{1}\right) / t\right)\right|^{4 / N} Q_{1}\left(\left(.-x_{1}\right) / t\right)+\left.\left.|t|^{-N / 2}|| t\right|^{-N / 2} Q_{2}\left(\left(.-x_{2}\right) / t\right)\right|^{4 / N}\right. \\
& \left.\cdot Q_{2}\left(\left(.-x_{2}\right) / t\right)\right\} \\
= & |t|^{-N}\left\{\left.\left\{\mid\left(Q_{1}\left(.-x_{1}\right) / t\right)+Q_{2}\left(\left(.-x_{2}\right) / t\right)\right)\right|^{4 / N}-\left|Q_{1}\left(\left(.-x_{1}\right) / t\right)\right|^{4 / N}\right\} \\
& \cdot Q_{1}\left(\left(.-x_{1}\right) / t\right)+\left\{\left|\left(Q_{1}\left(\left(.-x_{1}\right) / t\right)+Q_{2}\left(\left(.-x_{2}\right) / t\right)\right)\right|^{4 / N}-\left|Q_{2}\left(\left(.-x_{1}\right) / t\right)\right|^{4 / N}\right\} \\
& \left.\cdot Q_{2}\left(\left(.-x_{2}\right) / t\right)\right\} .
\end{aligned}
$$

Consider for example

$\left.\left.\left.B(x, t)=\left.|t|^{-N}\left\{\mid\left(Q_{1}\left(x-x_{1}\right) / t\right)+Q_{2}\left(\left(x-x_{2}\right) / t\right)\right)\right|^{4 / N}-\mid Q_{1}\left(x .-x_{1}\right) / t\right)\left.\right|^{4 / 5}\right\} Q_{1}\left(x-x_{1}\right) / t\right)$.

If $\left|\left(Q_{1}\left(\left(x-x_{1}\right) / t\right)|\geqq(1 / 2)| Q_{2}\left(\left(x-x_{2}\right) / t\right)\right)\right|$, then applying the mean value theorem (even if $4 / N-1 \leqq 0$ ), we obtain

$$
\begin{aligned}
|B(x, t)| \leqq c|t|^{-N}\left\{\left|Q_{1}\left(\left(x-x_{2}\right) / t\right)\right|^{(4 / N)-1}+\left|Q_{2}\left(\left(x-x_{1}\right) / t\right)\right|^{(4 / N)-1}\right\}\left|Q_{1}\left(\left(x-x_{1}\right) / t\right)\right| \\
\cdot\left|Q_{2}\left(\left(x-x_{2}\right) / t\right)\right| .
\end{aligned}
$$

If $\left.(1 / 2) \mid Q_{2}\left(\left(x-x_{2}\right) / t\right)\right)|\geqq|\left(Q_{1}\left(\left(x-x_{1}\right) / t\right) \mid\right.$, a direct estimation yields

$$
|B(x, t)| \leqq c|t|^{-N}\left\{\left|Q_{2}\left(\left(x-x_{1}\right) / t\right)\right|^{4 / N}\right\}\left|Q_{1}\left(\left(x-x_{1}\right) / t\right)\right| .
$$

Thus

$$
\|A\|_{L^{\tilde{\sigma}}} \leqq c|t|^{-N}\left\{\left\|Q_{1}\left(\left(.-x_{2}\right) / t\right)^{4 / N} Q_{2}\left(\left(.-x_{2}\right) / t\right)\right\|_{L^{\bar{\sigma}}}+\left\|Q_{2}\left(\left(.-x_{2}\right) / t\right)^{4 / N} Q_{1}\left(\left(.-x_{1}\right) / t\right)\right\|_{L^{\bar{\sigma}}}\right\} .
$$

We have from Lemma $4,\|A\|_{L^{\dot{\sigma}}} \leqq c|t|^{-N} e^{\left\{(-2 \theta /(\sigma t))\left|x_{1}-x_{2}\right|\right\}} \leqq c e^{\left\{(-\theta /(\sigma t))\left|x_{1}-x_{2}\right|\right\}}$ for $-1 \leqq t<0$ (with the same notations as the ones in the proof of Lemma 4) and part i) follows.

Part ii) is consequence of calculations similar to the ones done in part i). Part iii) follows from the proof of part i).

We now state and prove a fundamental inequality to obtain crucial a priori estimates for the proof of the theorem.

Lemma 6. Let $c_{1}>0$. For $\gamma$ large, there is a constant $\tau>0$ such that

$$
\forall t \in[0, \tau], \quad \int_{0}^{t}(t-s)^{-(1+2 / N)^{-1}} s^{-2(\sigma-2) / \sigma} e^{-(\gamma / s)} d s \leqq c_{1} e^{-(\gamma / t)} .
$$

Proof. The proof is somewhat technical. Showing that

$$
\int_{0}^{t}(t-s)^{-(1+2 / N)^{-1}} s^{-2(\sigma-2) / \sigma} e^{-(\gamma / s)} d s \leqq c_{1} e^{-\gamma / t}
$$

is equivalent to showing

$$
\int_{0}^{t}(t-s)^{-(1+2 / N)^{-1}} s^{-2(\sigma-2) / \sigma} e^{-(\gamma(t-s) / s t)} d s \leqq c_{1} .
$$


If we put $s=t u$, we obtain after some computation

$$
\int_{0}^{1}(1-u)^{-(1+2 / N)^{-1}} u^{-2(\sigma-2) / \sigma} e^{-(\gamma(1-u) / u t)} d u \leqq c_{1} t^{2 /(N+2)}
$$

On one hand, we have for $t$ near zero,

$$
\int_{0}^{1 / 2}(1-u)^{-(1+2 / N)^{-1}} u^{-2(\sigma-2) / \sigma} e^{-(\gamma(1-u) / u t)} d u \leqq c \int_{0}^{1 / 2} u^{-2(\sigma-2) / \sigma} e^{-(\gamma(1-u) / u t)} d u .
$$

Since $\forall u \in(0,1 / 2), 0<t \leqq 1$ and $\gamma>1$ we have $\gamma(1-u) / u t \geqq \gamma(1-u) / 2 u t+\gamma(1-u) / 2 u t \geqq$ $\gamma / 2 t+1 / 4 u$.

Thus

$$
\int_{0}^{1 / 2}(1-u)^{-(1+2 / N)^{-1}} u^{-2(\sigma-2) / \sigma} e^{-(\gamma(1-u) / u t)} d u \leqq c \int_{0}^{1 / 2} u^{-2(\sigma-2) / \sigma} e^{-1 / 4 u} e^{-(\gamma / 2 t)} d u \leqq c e^{-(\gamma / t 2)}
$$

(where $c$ is a constant which does not depend on $t$ ).

On the other hand, we have

$$
\int_{1 / 2}^{1}(1-u)^{-(1+2 / N)^{-1}} u^{-2(\sigma-2) / \sigma} e^{-(\gamma(1-u) / u t)} d u \leqq c \int_{1 / 2}^{1}(1-u)^{-(1+2 / N)^{-1}} e^{-(\gamma(1-u) / t)} d u
$$

(from the fact that $\forall u \in(1 / 2,1),(1-u) / u \geqq(1-u)$ ).

We remark that

$$
\begin{aligned}
c \int_{1 / 2}^{1}(1-u)^{-(1+2 / N)^{-1}} e^{-(\gamma(1-u) / t)} d u & =c \int_{0}^{1 / 2}(u)^{-(1+2 / N)^{-1 / 2}} e^{-(\gamma u / t)} d u \\
& =c \int_{0}^{1 / 2 t}(s)^{-(1+2 / N)^{-1}} e^{-(\gamma s)} d s\left\{t^{2 /(N+2)}\right\} \\
& \leqq\left\{c \int_{0}^{+\infty}(s)^{-(1+2 / N)^{-1}} e^{-(\gamma s)} d s\right\} t^{2 /(N+2)} \\
& \leqq c(\gamma) t^{2 /(N+2) \quad \text { (for } t \text { near zero) }}
\end{aligned}
$$

where $c(\gamma)$ goes to zero when $\gamma$ goes to infinity.

Using these two estimates, it is easy to conclude the proof of Lemma 6.

We are now in position to prove Proposition 1.

Proof of Proposition 1. Part i) follows from the fixed point formulation of Eq. ( $\varepsilon$ ) and a priori estimates (Lemmas 5 and 6).

For convenience, as before we denote the function

$$
\sum_{i=1}^{i=k}\left(|t|^{-N / 2} e^{-i / t+\left(i|x|^{2}\right) / 4 t} Q_{i}\left(\left(-x_{i}\right) / t\right)\right) \text { by } Q_{T}(t, x) .
$$

We recall that computations yield $\left\|Q_{T}(s)\right\|_{L^{\sigma}} \leqq \alpha /|s|^{2 / \sigma}$. The assumptions of the proposition imply that for $t<s \leqq-\varepsilon,\left\|u_{\varepsilon}(s)\right\|_{L^{\sigma}} \leqq(\alpha+1) /|s|^{2 / \sigma}$.

Since for $i=1, \ldots, k,|t|^{-N / 2} e^{(-i / t)+\left(i|x|^{2}\right) / 4 t} Q_{i}\left(\left(.-x_{i}\right) / t\right)$ is a solution of Eq. (1), we 
have

$$
\begin{aligned}
|t|^{-N / 2} & e^{(-i / t)+\left(i|x|^{2}\right) / 4 t} Q_{i}\left(\left(.-x_{i}\right) / t\right) \\
= & S(t+\varepsilon)\left\{\left(|\varepsilon|^{-N / 2} e^{i / \varepsilon-i|x|^{2} / 4 \varepsilon} Q_{i}\left(\left(.-x_{i}\right) /-\varepsilon\right)\right)\right\}+\int_{-\varepsilon}^{t} S(t-s)\left\{\mid\left(|s|^{-N / 2} e^{(-i / s)+\left(i|x|^{2}\right) / 4 s}\right.\right. \\
& \left.\left.\cdot Q_{i}\left(\left(.-x_{i}\right) / s\right)\right)\left.\right|^{4 / N}\left(|s|^{-N / 2} e^{(-i / s)+\left(i|x|^{2}\right) / 4 s} Q_{i}\left(\left(.-x_{i}\right) / s\right)\right)\right\} d s .
\end{aligned}
$$

\section{Hence}

$$
\begin{aligned}
& Q_{T}(t)=S(t+\varepsilon) Q_{T}(-\varepsilon)+i \int_{-\varepsilon}^{t} S(t-s)\left|Q_{T}(s)\right|^{4 / N} Q_{T}(s) d s-i \int_{-\varepsilon}^{t} S(t-s) \\
& \quad\left\{\left|Q_{T}(s)\right|^{4 / N} Q_{T}(s)-\left.\left.\sum_{i=1}^{i=k}|s|^{-N / 2} e^{(-i / s)+\left(i|x|^{2} / 4 s\right)}|| s\right|^{-N / 2} Q_{i}\left(\left(.-x_{i}\right) / s\right)\right|^{4 / N} Q_{i}\left(\left(.-x_{i}\right) / s\right)\right\} d s .
\end{aligned}
$$

On the other hand, since $u_{\varepsilon}(-\varepsilon)=Q_{T}(-\varepsilon)$ we have

$$
u_{\varepsilon}(t)=S(t+\varepsilon) Q_{T}(-\varepsilon)+i \int_{-\varepsilon}^{t} S(t-s)\left|u_{\varepsilon}(s)\right|^{4 / N} u_{\varepsilon}(s) d s
$$

This yields

$$
\begin{aligned}
& \left\{u_{\varepsilon}(t)-Q_{T}(t)\right\}=i \int_{-\epsilon}^{t} S(t-s)\left\{\left|u_{\varepsilon}(s)\right|^{4 / N} u_{\varepsilon}(s)-\left|Q_{T}(s)\right|^{4 / N} Q_{T}(s)\right\} d s-i \int_{-\varepsilon}^{t} S(t-s) \\
& \quad\left\{\left|Q_{T}(s)\right|^{4 / N} Q_{T}(s)-\left.\left.\left.\sum_{i=1}^{i=k}|s|^{-N / 2} e^{(-i / s)+\left(i|x|^{2} / 4 s\right)}|| t\right|^{-N}\right|^{2} Q_{i}\left(\left(.-x_{i}\right) / s\right)\right|^{4 / N} Q_{i}\left(\left(-x_{i}\right) / s\right)\right\} d s .
\end{aligned}
$$

Now taking the $L^{\sigma}$ norm of this equality and applying Lemmas 1,2 , we obtain

$$
\begin{aligned}
\left\|u_{\varepsilon}(t)-Q_{T}(t)\right\|_{L^{\sigma}} \leqq & \int_{\varepsilon}^{|t|} c|| t|-s|^{-1 /(1+2 / N)}\left\{\left\|u_{\varepsilon}(s)\right\|_{L^{\sigma}}^{4 / N}+\left\|Q_{T}(s)\right\|_{L^{\sigma}}^{4 / N}\right\} \\
& \cdot\left\|u_{\varepsilon}(s)-Q_{T}(s)\right\|_{L^{\sigma}} d s+c \int_{\varepsilon}^{|t|}|| t|-s|^{-1 /(1+2 / N)} \\
& \cdot \|\left|Q_{T}(s)\right|^{4 / N} Q_{T}(s)-\sum_{i=1}^{i=k}|s|^{-N / 2} e^{(-i / s)+\left(i|x|^{2 / 4} s\right)} \\
& \left.\cdot \|\left.\left. s\right|^{-N / 2} Q_{i}\left(\left(.-x_{i}\right) / s\right)\right|^{4 / N} Q_{i}\left(\left(\cdot-x_{i}\right) / s\right)\right) \|_{L^{\delta}} d s .
\end{aligned}
$$

Let us estimate the last term of this inequality. From Lemma 5 , we have for $t \in[-1,0)$,

$$
\begin{aligned}
& \int_{\varepsilon}^{|t|}|| t|-s|^{-1 /(1+2 / N)} \|\left|Q_{T}(s)\right|^{4 / N} Q_{T}(s)-\sum_{i=1}^{i=k}|s|^{-N / 2} e^{(-i / s)+\left(i|x|^{2 / 4 s}\right)} \\
& \cdot \|\left.\left.\left. s\right|^{-N / 2} Q_{i}\left(\left(.-x_{i}\right) / s\right)\right|^{4 / N} Q_{i}\left(\left(.-x_{i}\right) / s\right)\right|_{L^{\sigma}} d s \\
& \leqq c \int_{\varepsilon}^{|t|}|| t|-s|^{-1 /(1+2 / N)} e^{-\gamma /|s|} d s \leqq c e^{-\gamma /|t|} \int_{\varepsilon}^{|t|}|| t|-s|^{-1 /(1+2 / N)} d s \leqq c e^{-\gamma /|t|} .
\end{aligned}
$$


Hence the fact that for $s \in[t,-\varepsilon),\left\|u_{\varepsilon}(s)\right\|_{L^{\sigma}} \leqq(\alpha+1) /|s|^{2 / \sigma}$ implies that we have on $[t,-\varepsilon)$,

$$
\begin{gathered}
\left\|u_{\varepsilon}(\tau)-Q_{T}(\tau)\right\|_{L^{\sigma}} \leqq \int_{\varepsilon}^{|\tau|} c\left\|\tau|-s|^{-1 /(1+2 / N)}\left\{\left\|u_{\varepsilon}(s)\right\|_{L^{\sigma}}^{4 / N}+\left\|Q_{T}(s)\right\|_{L^{\sigma}}^{4 / N}\right\}\right\| u_{\varepsilon}(s)-Q_{T}(s) \|_{L^{\sigma}} \\
\cdot d s+c e^{-\gamma /|\tau|} \leqq c \int_{\varepsilon}^{|\tau|}\left\|\tau|-s|^{-1 /(1+2 / N)}|s|^{-2(\sigma-2) / \sigma}\right\| u_{\varepsilon}(s)-Q_{T^{\prime}}(s) \|_{L^{\sigma}} d s+c e^{-\gamma /|t|}
\end{gathered}
$$

We obtain a Gronwall type inequality. Unifortunately, the term $|\tau-s|^{-1 /(1+2 / N)}$ does not allow us to directly apply the Gronwall lemma. We obtain estimates on $\left\|u_{\varepsilon}(\tau)-Q_{T}(\tau)\right\|_{L^{\sigma}}$ by using a method of a priori estimates. Indeed, Lemma 6 implies for $\gamma$ large (that is from Lemma $5, \inf _{i \neq j}\left|x_{i}-x_{j}\right| \geqq \beta_{Q_{i}, k}$ ) the existence of $\varepsilon_{0}$ such that for $\varepsilon \in\left[0, \varepsilon_{0}\right)$, we have for

$$
\begin{aligned}
& -\forall t \in\left[-\varepsilon_{0},-\varepsilon\right], c e^{-\gamma /|t|}<(1 / 2) e^{-\gamma / 2|t|}, \\
& -\forall t \in\left[0, \varepsilon_{0}\right], c \int_{0}^{t}(t-s)^{(1+2 / N)^{-1}} s^{-2(\sigma-2) / \sigma} e^{-\gamma / 2 s} d s \leqq(1 / 2) e^{-\gamma / 2 t} .
\end{aligned}
$$

The fact that for all $-\varepsilon_{0}>t>-\varepsilon$,

$$
c \int_{\varepsilon}^{|\tau|}|| \tau|-s|^{-1 /(1+2 / N)}|s|^{-2(\sigma-2) / \sigma} e^{-\gamma / 2 s} d s+c e^{-\gamma /|t|} \leqq e^{-\gamma / 2|t|},
$$

and a priori estimate method implies that

$$
\text { for }-\varepsilon_{0}>t>-\varepsilon,\left\|u_{\varepsilon}(t)-Q_{T}(t)\right\|_{L^{\sigma}} \leqq e^{-\gamma / 2|t|}
$$

and part i) of Proposition 1 is proved.

We can easily check part ii) of Proposition 1 from part i) and a priori estimate techniques.

Assume that $\inf _{i \neq j}\left|x_{i}-x_{j}\right| \geqq \beta_{Q_{i}, k}$. With the same notations as the ones in part i), we can choose $\varepsilon_{0}$ such that for $t \in\left[-\varepsilon_{0}, 0\right), e^{-\gamma / 2|t|} \leqq 1 /|t|^{2 / \sigma}$. In this case, for $t \in\left[-\varepsilon_{0}, 0\right),\left\|u_{\varepsilon}(t)-Q_{T}(t)\right\|_{L^{\sigma}} \leqq e^{-\gamma / 2|t|}$ implies that

$$
\left\|u_{\varepsilon}(t)\right\|_{L^{\sigma}} \leqq e^{\gamma / 2|t|}+\left\|Q_{T}(t)\right\|_{L^{\sigma}} \leqq e^{-\gamma / 2|t|}+1 /|t|^{2 / \sigma} \leqq(\alpha+1) /|t|^{2 / \sigma} .
$$

We consider now, for $\varepsilon \in\left[-\varepsilon_{0}, 0\right)$, the first $t_{\varepsilon} \in(-1,-\varepsilon)$ such that $\left\|u_{\varepsilon}\left(t_{\varepsilon}\right)-Q_{T}\left(t_{\varepsilon}\right)\right\|_{L^{\sigma}}=e^{-\gamma / 2\left|t_{\sigma}\right|}$.

Applying part i), we obtain that $t_{\varepsilon} \leqq-\varepsilon_{0}$ and for $t \in\left[-\varepsilon_{0}, 0\right)$, we have $\left\|u_{\varepsilon}(t)-Q_{T}(t)\right\|_{L^{\sigma}} \leqq e^{-\gamma / 2|t|}$. This ends the proof of Proposition 1 .

\section{Compactness Results}

In this section, we assume that $\inf \left|x_{i}-x_{j}\right| \geqq \beta_{Q_{i}, k}$, where $\beta_{Q_{i}, k}$ is the constant defined in Proposition 1 (see Sect. 3). Therefore we can check from Sect. 3 that there are constants $\varepsilon_{0}>0, a>0, \gamma>0, K>0$, such that for $\varepsilon \in\left(0, \varepsilon_{0}\right]$, we have

$-\left\|u_{\varepsilon}(-a)\right\|_{L^{\sigma}} \leqq K$,

$-\left\|u_{\varepsilon}(t)-Q_{T}(t)\right\|_{L^{\sigma}} \leqq e^{-\gamma /|t|}$, for $t \in[-a,-\varepsilon)$. 
Our purpose is to have more information on the set $\left\{u_{\varepsilon}(-a)\right\}$, and in particular to have compactness results on this set in order to find a sequence $\varepsilon_{n} \rightarrow 0$ and a function $\varphi$ such that $u_{\varepsilon_{n}}(-a) \rightarrow \varphi$ in a suitable topology. Our main tools to obtain such estimates are to use the conservation laws of Eq. (1), the fact that $u_{\varepsilon}(-\varepsilon)=Q_{T}(-\varepsilon)$ and for $t$ near zero, the function $|t|^{-N / 2} e^{i / t+\left(i|x|^{2}\right) / 2 t} Q_{i}\left(\left(.-x_{i}\right) / t\right)$ is concentrated at the point $x_{i}$.

Lemma 7. Suppose $u_{\varepsilon}(-a)$ is defined for $\varepsilon \in\left(0, \varepsilon_{0}\right]$. Then

$$
\begin{aligned}
& -E\left(u_{\varepsilon}(-a)\right) \underset{\varepsilon \rightarrow 0}{\longrightarrow} \sum_{i=1}^{i=k} E\left(e^{i-i|x|^{2} / 4} Q_{i}\left(-\left(.-x_{i}\right)\right),\right. \\
& -\left\|u_{\varepsilon}(-a)\right\|_{L^{2}}^{2} \underset{\varepsilon \rightarrow 0}{\longrightarrow} \sum_{i=1}^{i=k}\left\|Q_{i}\right\|_{L^{2}}^{2}, \\
& -\int|x|^{2}\left|u_{\varepsilon}(-a)\right|^{2} \underset{\varepsilon \rightarrow 0}{\longrightarrow} \sum_{i=1}^{i=k} \int|x|^{2}\left|Q_{i}\left(\left(x-x_{i}\right) /(-a)\right)\right|^{2} .
\end{aligned}
$$

Proof. The conservation laws yield for all $\varepsilon \in\left(0, \varepsilon_{0}\right]$, and

$$
E\left(u_{\varepsilon}(-a)\right)=E\left(u_{\varepsilon}(-\varepsilon)\right)=E\left(Q_{T}(-\varepsilon)\right)=E\left\{\sum_{i=1}^{i=k}|\varepsilon|^{-N / 2} e^{i / \varepsilon-i|x|^{2} / 4 \varepsilon} Q_{i}\left(-\left(.-x_{i}\right) / \varepsilon\right)\right\},
$$

$$
\left\|u_{\varepsilon}(-a)\right\|_{L^{2}}=\left\|u_{\varepsilon}(-\varepsilon)\right\|_{L^{2}}=\left\|Q_{T}(-\varepsilon)\right\|_{L^{2}} .
$$

Lemma 5 implies that

$$
\left|E\left(Q_{T}(-\varepsilon)\right)-\left\{\sum_{i=1}^{i=k} E\left\{|\varepsilon|^{-N / 2} e^{i / \varepsilon-i|x|^{2} / 4 \varepsilon} Q_{i}\left(-\left(.-x_{i}\right) / \varepsilon\right)\right\}\right\}\right| \rightarrow 0
$$

and

as $\varepsilon$ goes to zero.

$$
\left|\left\|Q_{T}(-\varepsilon)\right\|_{L^{2}}^{2}-\sum_{i=1}^{i=k}\left\||\varepsilon|^{-N / 2} Q_{i}\left(-\left(.-x_{i}\right) / \varepsilon\right)\right\|_{L^{2}}^{2}\right| \rightarrow 0
$$

Since for a fixed $i$, the function $|t|^{-N / 2} e^{i / t+i|x|^{2} / 4 t} Q_{i}\left(-\left(.-x_{i}\right) / t\right)$ is a solution of Eq. (1), we have

and

$$
E\left\{|\varepsilon|^{-N / 2} e^{i / \varepsilon+\left(-i|x|^{2}\right) / 4 \varepsilon} Q_{i}\left(-\left(.-x_{i}\right) / \varepsilon\right)\right\}=E\left\{e^{i-i|x|^{2} / 4} Q_{i}\left(-\left(.-x_{i}\right)\right)\right\}
$$

$$
\left\||\varepsilon|^{-N / 2} Q_{i}\left(-\left(.-x_{i}\right) / \varepsilon\right)\right\|_{L^{2}}=\left\|Q_{i}\right\|_{L^{2}}
$$

We have

$$
E\left(u_{\varepsilon}(-a)\right) \underset{\varepsilon \rightarrow 0}{\longrightarrow} \sum_{i=1}^{i=k} E\left(e^{i-i|x|^{2} / 4} Q_{i}\left(-\left(.-x_{i}\right)\right), \quad \text { and } \quad\left\|u_{\varepsilon}(-a)\right\|_{L^{2}}^{2} \underset{\varepsilon \rightarrow 0}{\longrightarrow} \sum_{i=1}^{i=k}\left\|Q_{i}\right\|_{L^{2}}^{2}\right.
$$

The last conclusion follows from similar arguments and the conservation law (4).

We are now in position to prove the existence of a function $\varphi$ and a sequence $\varepsilon_{n} \rightarrow 0$ such that $u_{\varepsilon_{n}}(-a) \rightarrow \varphi$ in $L^{\sigma} \cap L^{2}$. Indeed, the following compactness lemma yields the result.

Lemma 8. Let $C$ be the set defined by $\left\{u ;\|u\|_{H^{1}} \leqq c\right.$ and $\left.\int|x|^{2}|u|^{2} \leqq c\right\}$ where $c$ is a positive constant. Then $C$ is a compact set in $L^{\sigma} \cap L^{2}$. 
Proof. We can easily check that $\forall \rho>0, \forall u \in C, \int_{|x| \geqq \rho}|u|^{2} \leqq c / \rho^{2}$. Since $\forall u \in C$, $\|u\|_{H^{1}} \leqq c$, from a classical argument, it follows that $C$ is a compact set in $L^{2}$. The Hölder and Sobolev inequalities $\left(\sigma \in\left[2,2^{*}\right)\right)$ yield the result.

Therefore we easily check

Proposition 2. Let $\beta_{Q_{i}, k}, a>0$ and $\gamma>0$ be the constants defined in Proposition 1. Assume that $\inf _{i \neq j}\left|x_{i}-x_{j}\right| \geqq \beta_{Q_{i}, k}$.

Then there are a sequence $\varepsilon_{n} \rightarrow 0$ and a function $\varphi \in H^{1}$ such that $u_{\varepsilon_{n}}(-a) \rightarrow \varphi$ in $L^{\sigma} \cap L^{2}$. In addition, we have $\|\varphi\|_{L^{2}}^{2}=\sum_{i=1}^{i=k}\left\|Q_{i}\right\|_{L^{2}}^{2}$.

Proof. It is consequence of Lemmas 7 and 8 . Let us consider the sequence $\varepsilon_{n}=1 / n$.

From Proposition 1, we check that for $n$ large, $u_{\varepsilon_{n}}(-a)$ is defined. Lemma 7 implies, for $n$ large, the existence of a constant $c>0$ such that $E\left(u_{\varepsilon_{n}}(-a)\right)<c$, $\left\|u_{\varepsilon_{n}}(-a)\right\|_{L^{2}}^{2}<c, \int|x|^{2}\left|u_{\varepsilon_{n}}(-a)\right|^{2}<c$. On the other hand, Proposition 1 yields that $\left\|u_{\varepsilon_{n}}(-a)-Q_{T}(-a)\right\|_{L^{\sigma}} \leqq e^{-\gamma / a}$. Thus $\left\|u_{\varepsilon_{n}}(-a)\right\|_{L^{\sigma}} \leqq c$ and from the energy estimates, $\left\|u_{\varepsilon_{n}}(-a)\right\|_{H^{1}} \leqq c$.

It follows from Lemma 8 that there is a $\varphi \in H^{1}$ such that $u_{\varepsilon_{n}}(-a) \rightarrow \varphi$ in $L^{\sigma} \cap L^{2}$ (the subsequence of $\left(\varepsilon_{n}\right)$ is also denoted $\left(\varepsilon_{n}\right)$ for convenience).

In addition, the fact that $\forall n,\left\|u_{\varepsilon_{n}}(-a)\right\|_{H^{1}}<c$ and $\int|x|^{2}\left|u_{\varepsilon_{n}}(-a)\right|^{2}<c$ implies that $\|\varphi\|_{H^{1}}<c, \int|x|^{2}|\varphi|^{2}<c$ and $\varphi \in H^{1}$.

Finally, $\left\|u_{\varepsilon_{n}}(-a)\right\|_{L^{2}}^{2} \rightarrow\|\varphi\|_{L^{2}}^{2}$ and $\left\|u_{\varepsilon_{n}}(-a)\right\|_{L^{2}}^{2}=\left\|u_{\varepsilon_{n}}\left(-\varepsilon_{n}\right)\right\|_{L^{2}}^{2} \rightarrow \sum_{i=1}^{i=k}\left\|Q_{i}\right\|_{L^{2}}^{2}$, when $n \rightarrow+\propto$. The uniqueness of the limit yields that $\|\varphi\|_{L^{2}}^{2}=\sum_{i=1}^{i=k}\|Q\|_{L^{2}}^{2}$. This concludes the proof of Proposition 2.

\section{Proof of the Theorem}

We may now prove the theorem. We use two steps. We first consider the case where $\inf \left|x_{i}-x_{j}\right| \geqq \beta_{Q_{i}, k}$, (where $\beta_{Q_{i}, k}$ is the constant defined in Proposition 1). In a second step, we prove the general case by reducing to the framework of the first step using rescaling arguments.

\section{Proof of the Theorem}

Step 1: $\inf _{i \neq j}\left|x_{i}-x_{j}\right| \geqq \beta_{Q_{1}, k}$

In this case, the conclusion follows from Propositions 1 and 2. Indeed, applying these proposition, we obtain the existence of a $a>0, \gamma>0$ and a sequence $\varepsilon_{n} \rightarrow 0$ such that

$-\left\|u_{\varepsilon_{n}}(t)-Q_{T}(t)\right\|_{L^{\sigma}} \leqq e^{-\gamma /|t|}$ on $\left[-a,-\varepsilon_{n}\right]$,

$-u_{\varepsilon_{n}}(-a) \rightarrow \varphi$ in $L^{\sigma} \cap L^{2}$ and $\varphi \in H^{1}$,

$-\|\varphi\|_{L^{2}}^{2}=\sum_{i=1}^{i=k}\left\|Q_{i}\right\|_{L^{2}}^{2}$ 
Let us denote by $u(t)$ the solution of the equation

$$
u(-a)=\varphi \quad \text { and } \quad i \partial u / \partial t=-\Delta u-|u|^{4 / N} u .
$$

We claim that $u(t)$ satisfies the conclusion of the theorem.

Let us check first that

$$
\begin{aligned}
\left\|u(t)-Q_{T}(t)\right\|_{L^{\sigma}} & =\left\|u(t, x)-\left\{\sum_{i=1}^{i=k}|t|^{-N / 2} e^{(-i / t)+\left(i|x|^{2}\right) / 4 t} Q_{i}\left(\left(x-x_{i}\right) / t\right)\right\}\right\|_{L^{\sigma}} \\
& \leqq e^{-\gamma /|t|} \text { on }[-a, 0] .
\end{aligned}
$$

Let $t \in[-a, 0)$. Since for $n$ large, $u_{\varepsilon_{n}}($.$) is defined on [-a, t]$, there is a constant $c$ such that $\forall s \in[-a, t], \forall n>n_{0},\left\|u_{\varepsilon_{n}}(s)\right\|_{H^{1}} \leqq c$, and $u_{\varepsilon_{n}}(-a) \rightarrow \varphi$ in $L^{\sigma} \cap L^{2}$. We have that for $s \in[-a, t], u_{\varepsilon_{n}}(s) \rightarrow u(s)$ in $L^{\sigma}$ (since the Cauchy problem is well-posed in the space $L^{\sigma}$ for initial data in $H^{1}$, we obtain this result using for example the techniques in [12]). Thus for $t \in[-a, 0), u_{\varepsilon_{n}}(t) \rightarrow u(t)$ as $n \rightarrow+\propto$ in $L^{\sigma}$. Finally, when $n$ goes to infinity in inequality (6), we obtain

$$
\begin{aligned}
\left\|u(t)-Q_{T}(t)\right\|_{L^{\sigma}} & =\left\|u(t, x)-\left\{\sum_{i=1}^{i=k}|t|^{-N / 2} e^{(-i / t)+\left(i|x|^{2}\right) / 4 t} Q_{i}\left(\left(x-x_{i}\right) / t\right)\right\}\right\|_{L^{\sigma}} \\
& \leqq e^{-\gamma /|t|} \text { on }[-a, 0] .
\end{aligned}
$$

We claim now that the conclusions of the theorem are consequences of this inequality.

Let us show that $u(t)$ blows up at time zero and that the blow-up set in $L^{2+4 / N}$, $B_{L^{2+4 / N}}=\left\{x_{1}, \ldots, x_{k}\right\}$. The inequality (7) implies that for $t \in[-a, 0),-e^{-\gamma /|t|}+$ $c /|t|^{2 / \sigma} \leqq\|u(t)\|_{L^{\sigma}} \leqq e^{-\gamma /|t|}+c /|t|^{2 / \sigma}$. Therefore $u(t)$ is defined on $[-a, 0)$ and blows up at time zero.

In the same way, we have $\forall x, \forall R>0$,

$$
-e^{-\gamma /|t|} \leqq\|u(t)\|_{L^{\sigma}(B(x, R))}-\left\|Q_{T^{\prime}}(t)\right\|_{L^{\sigma}(B(x, R))} \leqq e^{-\gamma /|t|} .
$$

If $x \notin\left\{x_{1}, \ldots, x_{k}\right\}$, there is an $R>0$ such that $\left\{x_{1}, \ldots, x_{k}\right\} \notin \bar{B}(x, R)$. Lemma 4 implies then that there is a constant $c>0$ such that

$\forall i, \forall t,\left\||t|^{-N / 2} e^{(i / t)+\left(i|x|^{2}\right) / 4 t} Q_{i}\left(\left(.-x_{i}\right) / t\right)\right\|_{L^{\sigma}(B(x, R))} \leqq c$ and inequality (8) yields that $\|u(t)\|_{L^{\sigma}(B(x, R))} \leqq c$ (where $c$ does not depend on $\left.t\right)$. Therefore $x$ is not a blow-up point.

If $x=x_{i}$ for a $i \in\{1, \ldots, k\}$, then for all $R<\beta_{Q_{i}, k}$, we have

$$
\left\||t|^{-N / 2} e^{(-i / t)+\left(i|x|^{2}\right) / 4 t} Q_{i}\left(\left(.-x_{i}\right) / t\right)\right\|_{L^{\sigma}(B(x, R))} \rightarrow+\propto,
$$

and for $j \neq i,\left\||t|^{-N / 2} e^{(-i / t)+\left(i|x|^{2}\right) / 4 t} Q_{j}\left(\left(.-x_{j}\right) / t\right)\right\|_{L^{\sigma}(B(x, R))} \rightarrow 0$.

The inequality (8) implies that $\|u(t)\|_{L^{\sigma}(B(x, R))} \rightarrow+\propto$ and $x$ is a blow-up point.

In conclusion, $u(t)$ blows-up in the norm $L^{2+4 / N}$ at exactly $\left\{x_{1}, \ldots, x_{k}\right\}$.

Our aim now is to prove that the blow-up points in $H^{1}$ are also exactly $\left\{x_{1}, \ldots, x_{k}\right\}$. We want to check the existence of a constant $K$ such that

$$
\left\|\nabla\left\{u(t)-Q_{T}(t)\right\}\right\|_{L^{2}} \leqq K \quad \text { for } \quad t \in[-a, 0) .
$$


We have

$$
\left\|\nabla\left\{u(t)-Q_{T}(t)\right\}\right\|_{L^{2}}^{2}=\|\nabla u(t)\|_{L^{2}}^{2}+\left\|\nabla Q_{T}(t)\right\|_{L^{2}}^{2}-2 \operatorname{Re} \int \nabla \bar{u}(t) \nabla Q_{T}(t) .
$$

On one hand,

$$
\begin{aligned}
\operatorname{Re} \int \nabla \bar{u}(t) \nabla Q_{T}(t) & =-\operatorname{Re} \int \bar{u}(t) \Delta Q_{T}(t) \\
& =-\operatorname{Re} \int \bar{u}(t) \Delta\left\{\sum_{i=1}^{i=k}|t|^{-N / 2} e^{(-i / t)+\left(i|x|^{2}\right) / 4 t} Q_{i}\left(\left(.-x_{i}\right) / t\right)\right\} .
\end{aligned}
$$

Then using Eq. (5), the inequality (7) and Lemmas 3, 4 and 5, we obtain after some calculations that there exists a constant $K$ such that $\mid \operatorname{Re} \int \nabla \bar{u}(t) \nabla Q_{T}(t)-$ $\left\|\nabla Q_{T}(t)\right\|_{L^{2}}^{2} \mid \leqq K$. On the other hand, similar calculations, Lemma 5 , and the conservation of the energy yield that $\left|\|\nabla u(t)\|_{L^{2}}^{2}-\left\|\nabla Q_{T}(t)\right\|_{L^{2}}^{2}\right| \leqq K$ (where $K$ does not depend on $t$ ).

Finally, we prove that there is a constant $K$ such that $\left\|\nabla\left\{u(t)-Q_{T}(t)\right\}\right\|_{L^{2}} \leqq K$ on $[-a, 0)$. We then conclude as before.

We claim now that for $i=1, \ldots, k$, and small $R>0, \lim _{t \rightarrow T}\|u(t)\|_{L^{2}\left(B\left(x_{i}, R\right)\right)}=\left\|Q_{i}\right\|_{L^{2}}$, and $\lim _{t \rightarrow T}\|u(t)\|_{L^{2}}\left(\mathbb{R}^{N} \backslash \bigcup_{i=1, \ldots, k} B\left(x_{i}, R\right)\right)=0$, as a consequence of the inequality (7) and the conservation of mass of the solution of Eq. (1) ((2)).

On one hand, the Hölder inequality yields, for fixed $i$ and $R$, that

$$
\begin{array}{r}
\left\|u(t)-Q_{T}(t)\right\|_{L^{2}\left(B\left(x_{i}, R\right)\right)} \leqq\left\|u(t)-Q_{T}(t)\right\|_{L^{\sigma}\left(B\left(x_{i}, R\right)\right)}\|1\|_{L^{r}\left(B\left(x_{i}, R\right)\right)} \text { on }[-a, 0) \\
\text { (where } r=2 \sigma /(\sigma-2)) .
\end{array}
$$

It follows from (7) that $\left\|u(t)-Q_{T}(t)\right\|_{L^{2}\left(B\left(x_{i}, R\right)\right)} \leqq c e^{-\gamma /|t|}$ on $[-a, 0]$. Lemma 4 implies that for $R \leqq \beta_{Q_{i}, k} / 2$,

$$
\left\|Q_{T^{\prime}}(t)\right\|_{L^{2}\left(B\left(x_{i}, R\right)\right)} \rightarrow\left\|Q_{i}\right\|_{L^{2}}^{2} \text { as } t \rightarrow 0 \text { and }\|u(t)\|_{L^{2}\left(B\left(x_{i}, R\right)\right)}^{2} \rightarrow\left\|Q_{i}\right\|_{L^{2}}^{2} \text { as } t \rightarrow 0 .
$$

On the other hand, from the conservation of the $L^{2}$ norm, we can check that for $i=1, \ldots, k$, and all $\left.R>0, \lim _{t \rightarrow T}\|u(t)\|_{L^{2}\left(\mathbb{R}^{N} \backslash\right.} \bigcup_{i=1, \ldots, k} B\left(x_{i}, R\right)\right)=0$ and the theorem is proved in the case where $\inf _{i \neq j}\left|x_{i}-x_{j}\right| \geqq \beta_{Q_{i}, k}$.

Step 2: $\inf _{i \neq j}\left|x_{i}-x_{j}\right| \leqq \beta_{Q_{i}, k}$.

We derived the result in the general case using rescaling arguments. Let us consider $\omega>0$ such that $\omega \cdot \inf _{i \neq j}\left|x_{i}-x_{j}\right| \geqq \beta_{Q_{i}, k}$. Applying step 1 , we obtain $a>0$ and $\varphi \in H^{1}$ such that $u(-a)=\varphi$ and

$-i \partial u / \partial t=-\Delta u-|u|^{4 / N} u$

$-u(t)$ blows up at time zero,

- the sets of blow-up points in $L^{2+4 / N}$ and in $H^{1}$ is $\left\{\omega x_{1}, \omega x_{2}, \ldots, \omega x_{k}\right\}$,

- and for $i=1, \ldots, k$ and small $R>0, \lim _{t \rightarrow 0}\|u(t)\|_{L^{2}\left(B\left(\omega x_{i}, R\right)\right)}=\left\|Q_{i}\right\|_{L^{2}}$, 
-for all $\left.R>0, \lim _{t \rightarrow 0}\|u(t)\|_{L^{2}\left(\mathbb{R}^{N} \backslash\right.} \bigcup_{i=1, \ldots, k} B\left(\omega x_{i}, R\right)\right)=0$,

— there exists $\gamma>0$ such that

$$
\left\|u(t)-\left\{\sum_{i=1}^{i=k}|t|^{-N / 2} e^{(-i / t)+\left(i|x|^{2}\right) / 4 t} Q_{i}\left(\left(.-\omega x_{i}\right) / t\right)\right\}\right\|_{L^{\sigma}} \leqq e^{-\gamma /|t|} \text { on }[-a, 0) .
$$

Considering now $v(t, x)=u\left(t \omega^{2}, x \omega\right) \omega^{N / 2}$, we obtain a function such that

$-i \partial v / \partial t=-\Delta v-|v|^{4 / N} v$

$-v(t)$ blows up at time zero,

- the sets of blow-up points in $L^{2+4 / N}$ and in $H^{1}$ is $\left\{x_{1}, x_{2}, \ldots, x_{k}\right\}$,

-and for $i=1, \ldots, k$ and small $R>0, \lim _{t \rightarrow T}\|v(t)\|_{L^{2}\left(B\left(x_{i}, R\right)\right)}=\left\|Q_{i}\right\|_{L^{2}}$,

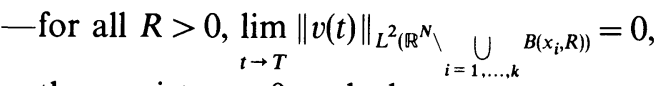

- there exists $\gamma>0$ such that

$$
\left\|v(t, x)-\left\{\sum_{i=1}^{i=k}|t \omega|^{-N / 2} e^{\left(-i / t \omega^{2}\right)+\left(i|x|^{2}\right) / 4 t} Q_{i}\left(\left(x-x_{i}\right) / t \omega\right)\right\}\right\|_{L^{\sigma}} \leqq e^{-\gamma /|t|} \text { on }[-a, 0) \text {. }
$$

This concludes the proof of the theorem.

Remark 7. Of course to apply step 1, instead of rescaling on the points $x_{1}, \ldots, x_{k}$, we could rescale the functions $Q_{i}$.

\section{References}

1. Berestycki, H., Lions, P. L., Peletier, L. A.: An ODE approach to the existence of positive solutions for semilinear problems in $\mathbb{R}^{N}$. Ind. Univ. Math. J. 30, 141-157 (1981)

2. Berestycki, H., Lions, P. L.: Existence d'ondes solitaires dans des problèmes nonlinéaires du type Klein-Gordon, C. R. Paris 287, 503-506 (1978); 288, 395-398 (1979)

3. Berestycki, H., Cazenave, T.: Instabilité des états stationnaies dans les équations de Schrödinger et de Klein-Gordon non-linéaires. C. R. Paris 293, 489-492 (1981)

4. Ginibre, J., Velo, G.: On a class of nonlinear Schrödinger equations. I: The Cauchy problem, J. Funct. Anal. 32, 1-32 (1979)

5. Ginibre, J., Velo, G.: The global Cauchy problem for the nonlinear Schrödinger equation revisited, Ann. Inst. Henri Poincaré, Physique Théorique 4, 309-327 (1985)

6. Glassey, R. T.: On the blowing-up of solutions to the Cauchy problem for the nonlinear Schrödinger equation. J. Math. Phys. 18, 1794-1797 (1977)

7. Kato, T.: On nonlinear Schrödinger equations. Ann. Inst. Henri Poincaré. Phys. Théorique 46, $113-129$ (1987)

8. Landman, M., Papanicolaou, G. C., Sulem, C., Sulem, P. L.: Rate of blow-up for solutions of the nonlinear Schrödinger equation in critical dimension. Phys. Review A (to appear)

9. Lemesurier, B., Papanicolaou, G., Sulem, C., Sulem, P. L.: The focusing singularity of the nonlinear Schrödinger equation, preprint

10. Chen, X., Matano, H.: Convergence, asymptotic periodicity, and finite-point blow-up in one dimensional semilinear heat equations. J. Diff. Equ. 78, 160-190 (1989)

11. Merle, F.: Limit of the solution of the nonlinear Schrödinger equation at the blow-up time. J. Funct. Analysis (to appear)

12. Merle, F.: Sur la dépendance continue de la solution de l'équation de Schrödinger non linéaire près du temps d'explosion. C. R. Paris 16, 479-482 (1987) 
13. Merle, F., Tsutsumi, Y.: $L^{2}$-concentration of blow-up solutions for the nonlinear Schrödinger equation with the critical power nonlinearity, preprint

13'. Schoen, R. M.: The existence of weak solutions with prescribed singular behavior for a conformally invariant scalar equation. Commun. Pure Appl. Math. V16, 3, 317-392 (1988)

14. Tsutsumi, Y.: $L^{2}$-solutions for nonlinear Schrödinger equations and nonlinear groups. Funk. Ekva. 30, 115-125 (1987)

15. Weinstein, M. I.: Nonlinear Schrödinger equations and sharp interpolation estimates. Commun. Math. Phys. 87, 567-576 (1983)

16. Weinstein, M. I.: On the structure and formation of singularities in solutions to the nonlinear dispersive evolution equations. commun. Partial Differential Equations 11, 545-565 (1986)

17. Zakharov, V. E., Sobolev, V. V., Synach, V. S.: Character of the singularity and stochastic phenomena in self-focusing, Zh. Eksp. Teor. Fiz., Pis'ma Red 14, 390-393 (1971)

Communicated by R. Spencer

Received March 16, 1989; in revised form July 19, 1989 